\title{
Mortality in Graves' orbitopathy is increased and influenced by gender, age and pre-existing morbidity: a nationwide Danish register study
}

\author{
Charlotte F Schwensen ${ }^{1}$, Frans Brandt' ${ }^{2}$, Laszlo Hegedüs ${ }^{1}$ and Thomas H Brix ${ }^{1}$ \\ 'Department of Endocrinology and Metabolism, Odense University Hospital, Odense, Denmark and ${ }^{2}$ Department \\ of Internal Medicine, Hospital of Southern Jutland, Sønderborg, Denmark
}

\author{
Correspondence \\ should be addressed \\ to F Brandt \\ Email \\ frans.brandt.kristensen@ \\ rsyd.dk
}

\begin{abstract}
Introduction: It is unclear whether the excess mortality associated with Graves' disease differs between individuals with Graves' orbitopathy (GO) or without (GD).

Subjects and methods: A nationwide, register-based cohort study in which all adult Danes diagnosed with GD ( $n=28461)$ and GO ( $n=3965)$ between 1995 and 2012 were matched for age and gender with four control subjects. Median follow-up time was 7.9 years (range 0 -17.5). Mortality risk in GO patients compared to the control population and compared to GD patients was calculated using Cox regression analyses, adjusting for pre-existing morbidity using the Charlson score.

Results: Adjusted mortality in Graves' disease overall (GD + GO) was significantly increased compared to that in the background population ( $H R=1.18$ (95\% confidence interval: 1.15-1.21)). In GD and GO separately, adjusted mortality was also significantly higher than that in their respective control populations (HR: 1.19 (1.16-1.22) and HR: 1.23 (1.121.35) respectively). However, mortality in GO compared to that in GD was decreased (HR: 0.64 (0.59-0.69)), although this difference attenuated after adjustment for pre-existing morbidity, age and gender. Both GD and GO males had a significantly higher mortality than those in females. For GO, but not for GD, mortality risk was the highest in the youngest and decreased with increasing age.

Conclusions: GD and GO were associated with increased mortality, especially in males. In GO, but not in GD patients, there was an inverse relationship between age and mortality. Surprisingly, and in need of further study, mortality was not higher in GO than that in GD individuals.
\end{abstract}

European Journal of

Endocrinology

(2017) 176, 669-676

\section{Introduction}

Graves' disease is the most common cause of hyperthyroidism with a lifetime risk of $3 \%$ and $0.5 \%$ in women and men respectively (1). The diagnosis is preceded by excess non-thyroidal morbidity and followed by increased comorbidity, somatic (2) as well as psychiatric (3). In addition, subjects with hyperthyroidism have an increased risk of long-term sickness absence, unemployment, work disability, loss of labour market income and disability pensioning (4). Therefore, it is no surprise that a recent meta-analysis, albeit based on studies with a number of shortcomings, found an excess mortality of approximately $20 \%$ in hyperthyroid subjects (5). Subsequently, in a random 5\% sample of the Danish population, we have demonstrated a $42 \%$ increase in mortality in patients diagnosed with Graves' disease as compared to the background population (6). However, in
() 2017 European Society of Endocrinology Printed in Great Britain
Published by Bioscientifica Ltd. 
that study, mainly due to inadequate power, we were unable to differentiate between patients with and without Graves' orbitopathy.

Graves' orbitopathy is the most common extrathyroidal manifestation of Graves' disease and has been reported to affect 5-20\% of patients, out of whom approximately $5 \%$ are severely affected and primarily need immunosuppression with corticosteroids $(7,8)$. Studies with limited numbers have suggested that the development of Graves' orbitopathy negatively influences daily life. Thus, patients have longer sick leave (9) and significantly reduced quality of life (10). Other small studies have found it associated with increased psychosocial morbidity (11). Of importance, the treatment modalities for Graves' orbitopathy, such as high-dose glucocorticoids, retrobulbar irradiation or surgery, may cause severe side effects. In fact, each of these treatment options has side effect profiles that may inflict physical and psychological morbidity and thereby increase mortality. Despite the previously mentioned considerations, we lack insight into whether the mortality risk associated with Graves' orbitopathy differs from that in Graves' disease without eye involvement. Accepting that Graves' orbitopathy is clinically the gravest manifestation of hyperthyroidism, we hypothesised that prognosis with respect to mortality in Graves' orbitopathy compared to Graves' disease without orbitopathy would be poorer.

Denmark has a long tradition for storing information on its citizens in nationwide registers, mainly for administrative use. This has resulted in various databases holding e.g., information on demographics, health and mortality (12). Linking these databases provides a unique opportunity for large-scale, population-based investigations. We sought to address (i) whether the mortality in Graves' orbitopathy (GO) differs from that in the background population, (ii) whether there is a genderand/or age-related difference in mortality in patients with Graves' disease without orbitopathy (GD) and GO and (iii) whether the mortality differs between GO and GD.

\section{Subjects and methods}

This is a register-based observational cohort study utilising the databases described below, all of which are hosted at Statistics Denmark. By means of a unique 10-digit personal identification number, record linkage on an individual level between them is possible. Due to the register-based design, no informed consent was required.

\section{Data sources}

The Danish Civil Registration System (DCRS) contains information on demographics, vital status, date of death and residence of all persons living or having lived in Denmark (13).

The Danish National Patient Register (DNPR) (14) contains information on all diagnoses from somatic hospital wards and/or outpatient admissions since 1995. Registrations of diagnoses are based on the Danish edition of the International Classification of Diseases, using the 10th revision (ICD-10) from 1994 and up until now.

The Danish National Prescription Registry (DNPrR) holds information on prescriptions of drugs dispensed from Danish pharmacies since 1995 (15). Medical products are coded according to the Anatomic Therapeutic Chemical (ATC) Classification System. The registry covers information on the date of dispensing, ATC code, strength and quantity in defined daily doses. Law requires that Danish pharmacies register all prescriptions dispensed at an individual level to ensure partial reimbursement for drug expenses from the healthcare system. It follows that DNPrR constitutes a highly valid source of information for our purpose (2).

\section{Operational definitions}

Utilising the DNPR, subjects with GO were defined by the ICD-10 codes H052 or H062, and subjects with GD by the ICD-10 code E050. Control subjects were defined by no history of either hypothyroidism or hyperthyroidism or GO. That is, subjects without any thyroid-related ICD-10 codes in DNPR and no evidence of treatment with thyroidrelated drugs (no ATC codes for dispensed prescriptions of thyroid hormone (H03A) or antithyroid drugs (H03B) in DNPrR).

\section{Study population}

To ensure inclusion of incident cases only, we used the first 6 months of 1995 as a washout period and thus excluded all cases appearing in DNPR with a thyroid diagnosis during this period. Figure 1 shows the selection of the study population. 3965 subjects with GO and 28461 with GD were identified. Each of these subjects was randomly matched for age and gender with four control subjects. 


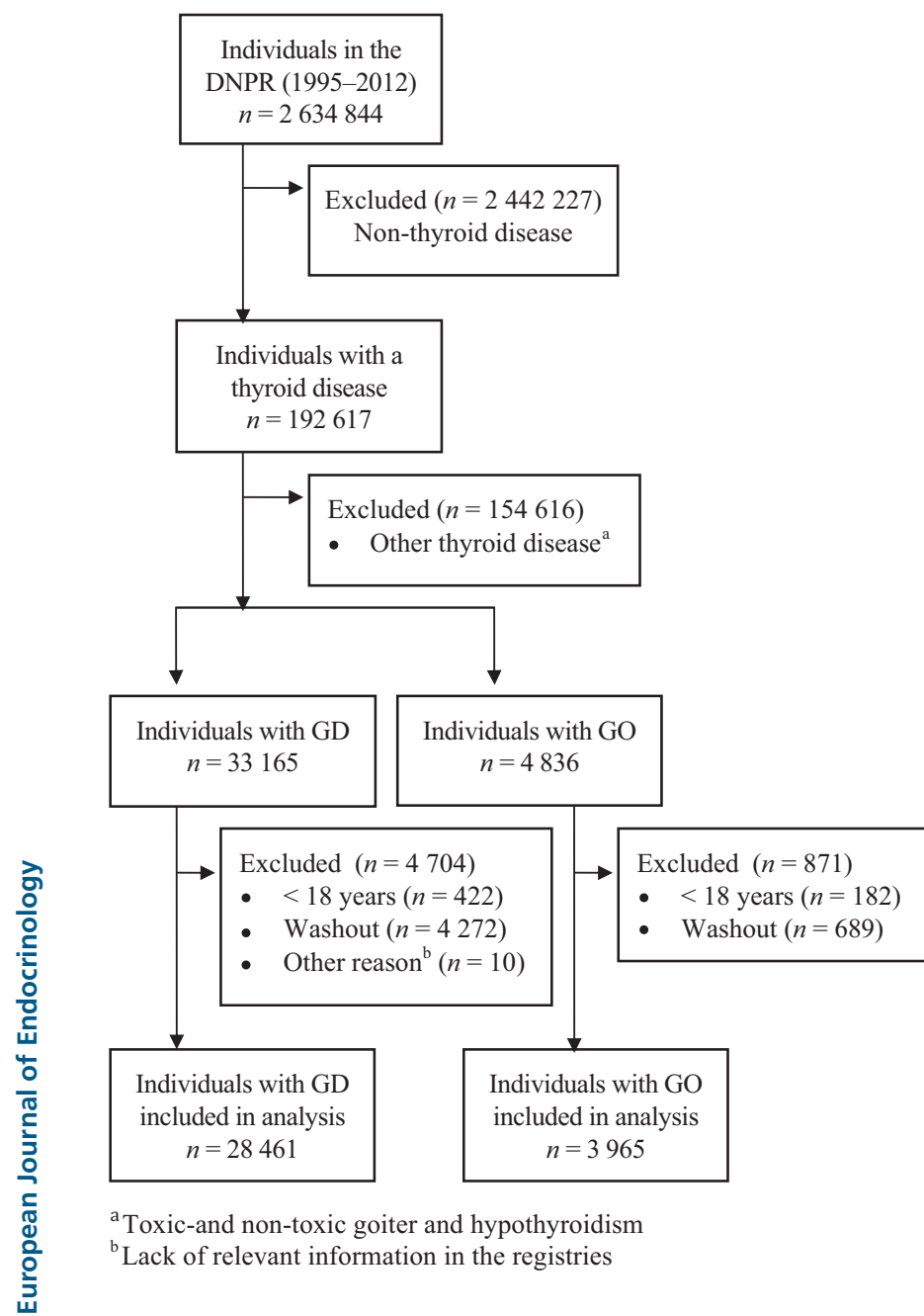

Figure 1

Selection of individuals with Graves' disease without orbitopathy (GD) and with Graves' orbitopathy (GO).

\section{Mortality}

Information on the date of death was obtained from the DCRS.

\section{Morbidity}

In order to adjust for confounding, we used the Charlson score (CS) to control for pre-existing morbidity. The CS includes 19 disease categories; each assigned a score from 1 to 6 , depending on its severity. The CS is the sum of the scores for all conditions on an individual level. The CS has been validated for different morbidities including benign disorders (16) and has been used in a number of publications investigating the long-term consequences of thyroid disease $(2,3,6)$. In the present study, we based the CS primarily on information from the DNPR. However, in

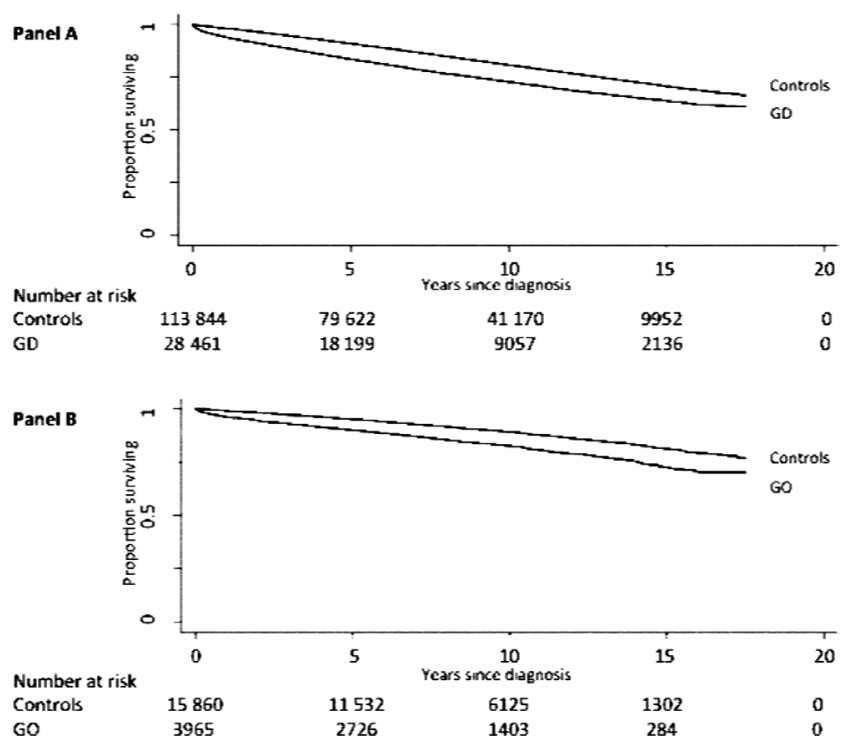

Figure 2

Kaplan-Meier survival estimates for Graves' disease without orbitopathy (GD, panel A), and for Graves' orbitopathy (GO, panel B), compared to their respective controls.

Denmark, many patients with diabetes, cardiovascular diseases and lung diseases do not get diagnosed and treated in a hospital setting but are solely handled in primary care. As a consequence, we also based the CS on information from the DNPrR for these disease categories. Thus, we classified all users of anti-diabetics (ATC code: A10), cardiovascular-related drugs (B01, C01, C03, C07, C08, C09 or N02) and drugs for obstructive airway diseases (R03), registered in the DNPrR, as having diabetes, cardiovascular diseases or lung diseases respectively.

\section{Statistical analysis}

Group frequencies were compared with the Pearson $\chi^{2}$ test, whereas group means and medians were compared by a $t$-test and the Mann-Whitney test respectively. We compared mortality rates in GO with those in the control subjects and the GD subjects and evaluated the effect of gender and age stratification. Mortality risk was evaluated by Cox's proportional hazard model, with time since diagnosis as the underlying time variable. Accordingly, person-years of follow-up were accumulated from the date of diagnosis and terminated at the date of death, emigration or the end of follow-up (31 December 2012), whichever came first.

In the analyses comparing mortality in GO and GD, immortal time bias could be present (17). Based on the assumption that the GO diagnosis often follows the GD diagnosis, subjects with GO are immortal in the period 
Table 1 Basic characteristics.

\begin{tabular}{|c|c|c|c|}
\hline & \multicolumn{3}{|c|}{ Graves' disease without orbitopathy (GD) } \\
\hline & Cases & Controls & $P$-value \\
\hline Number & 28461 & 113844 & - \\
\hline $\begin{array}{l}\text { Median age at } \\
\text { diagnosis (years) }\end{array}$ & $55(18-102)$ & $55(18-102)$ & - \\
\hline Female (\%) & 82 & 82 & - \\
\hline Number with $\mathrm{CS}^{\mathrm{a}}=0$ & $7056(25 \%)$ & $50116(44 \%)$ & $<0.001$ \\
\hline Number with $\mathrm{CS}=1$ & $11967(42 \%)$ & $39140(34 \%)$ & $<0.001$ \\
\hline Number with $\mathrm{CS}=2$ & $5586(20 \%)$ & $15372(14 \%)$ & $<0.001$ \\
\hline Number with $\mathrm{CS}>2$ & $3852(14 \%)$ & $9216(8 \%)$ & $<0.001$ \\
\hline Median CS & 1 & 1 & $<0.001$ \\
\hline
\end{tabular}

\begin{tabular}{|c|c|c|c|}
\hline \multicolumn{3}{|c|}{ Graves' orbitopathy (GO) } & GO vs GD \\
\hline Cases & Controls & $P$-value & $P$-value \\
\hline 3965 & 15860 & - & - \\
\hline 52 (18-98) & 52 (18-98) & - & $<0.001$ \\
\hline 73 & 73 & - & $<0.001$ \\
\hline $900(23 \%)$ & $7502(47 \%)$ & $<0.001$ & 0.004 \\
\hline $1773(45 \%)$ & $5301(33 \%)$ & $<0.001$ & 0.001 \\
\hline $752(19 \%)$ & $2039(13 \%)$ & $<0.001$ & 0.326 \\
\hline $540(13 \%)$ & $1018(7 \%)$ & $<0.001$ & 0.884 \\
\hline 1 & 1 & $<0.001$ & 0.248 \\
\hline
\end{tabular}

between debut of GD and the GO diagnosis. To minimise this bias, analyses comparing mortality rates in GD and GO individuals were repeated after excluding GD individuals dead or lost to follow-up within 6 months after the thyroid diagnosis. The validity of the proportional hazards assumption was evaluated by the inspection of Schoenfeld residuals vs follow-up time (no significant associations were present).

Significant differences were defined as a $P$ value $<0.05$, using two-tailed tests. All analyses were conducted using STATA, version 14.1 (2015; Statacorp).

\section{Results}

\section{Basic characteristics}

Table 1 summarises the basic characteristics of cases identified with GD or GO and their control subjects. Both GD and GO subjects had a significantly higher level of pre-existing morbidity than did their respective controls $(P<0.001)$. In contrast, there was no statistically significant difference in the burden of pre-existing morbidity (evaluated by the CS) between the GD and the GO cases $(P=0.248)$.

\section{Mortality analyses}

The hazard ratio (HR) for mortality in individuals with Graves' disease overall (both GD and GO), compared to the control population was significantly increased (HR: 1.53 (CI: 1.49-1.57); $P<0.001$ ). This persisted, although attenuated after adjustment for pre-existing morbidity (HR: 1.18 (1.15-1.21); $P<0.001)$ (Table 2).

Looking at individuals with GD and GO separately the survival curves are shown in Figure 2. The mortality risk in both groups was significantly higher than that in their respective control populations (HR: 1.19 (1.161.22) and HR: $1.23(1.12-1.35)$ respectively; $P<0.001)$ (Table 2). To further explore the impact of morbidity on the excess mortality in both GD and GO, we limited the analyses to subjects without any pre-existing morbidity, defined by $C S=0$. This yielded essentially similar results

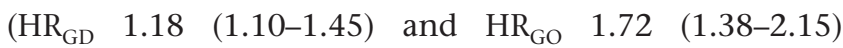
respectively; $P<0.001)$.

Table 2 Hazard ratio of mortality in Graves' disease overall (GD + GO), Graves' disease without orbitopathy (GD) and Graves' orbitopathy $(\mathrm{GO})$ compared to their respective control populations.

\begin{tabular}{|c|c|c|c|c|c|c|c|}
\hline \multirow{2}{*}{\multicolumn{2}{|c|}{ Study population }} & \multicolumn{2}{|c|}{ Number } & \multicolumn{4}{|c|}{ Hazard ratio } \\
\hline & & Cases & Controls & Non-adjusted & $P$ value & Adjusted $^{a}$ & $P$ value \\
\hline \multirow[t]{3}{*}{$\mathrm{GD}+\mathrm{GO}$} & All & 32426 & 129704 & $1.53(1.49-1.57)$ & $<0.001$ & $1.18(1.15-1.21)$ & $<0.001$ \\
\hline & Male & 6247 & 24988 & $1.83(1.73-1.93)$ & $<0.001$ & $1.34(1.27-1.41)$ & $<0.001$ \\
\hline & Female & 26179 & 104716 & $1.46(1.42-1.51)$ & $<0.001$ & $1.14(1.10-1.17)$ & $<0.001$ \\
\hline \multirow[t]{3}{*}{ GD } & All & 28461 & 113844 & $1.53(1.49-1.58)$ & $<0.001$ & $1.19(1.16-1.22)$ & $<0.001$ \\
\hline & Male & 5193 & 20772 & $1.79(1.69-1.90)$ & $<0.001$ & $1.32(1.25-1.40)$ & $<0.001$ \\
\hline & Female & 23268 & 93072 & $1.48(1.43-1.52)$ & $<0.001$ & $1.16(1.12-1.19)$ & $<0.001$ \\
\hline \multirow[t]{3}{*}{ GO } & All & 3965 & 15860 & $1.71(1.56-1.87)$ & $<0.001$ & $1.23(1.12-1.35)$ & $<0.001$ \\
\hline & Male & 1054 & 4216 & $2.21(1.90-2.57)$ & $<0.001$ & $1.52(1.30-1.78)$ & $<0.001$ \\
\hline & Female & 2911 & 11644 & $1.50(1.34-1.69)$ & $<0.001$ & $1.10(0.98-1.23)$ & 0.119 \\
\hline
\end{tabular}

${ }^{a}$ Adjusted for morbidity prior to the thyroid diagnosis using Charlson score. 
Table 3 Five year mortality risk in Graves' disease without orbitopathy (GD) and Graves' orbitopathy (GO), stratified for decades of age.

\begin{tabular}{|c|c|c|c|}
\hline \multirow[b]{2}{*}{ Study population } & \multirow[b]{2}{*}{ Age } & \multicolumn{2}{|r|}{ Cases } \\
\hline & & Number & $\begin{array}{c}5 \text {-year mortality risk (\%) } \\
\text { (number) }\end{array}$ \\
\hline \multirow[t]{5}{*}{ GD } & $<40$ & 6996 & $0.4(27)$ \\
\hline & $40-49$ & 4510 & $1.5(70)$ \\
\hline & $50-59$ & 4886 & $4.9(241)$ \\
\hline & $60-69$ & 4229 & $13.4(565)$ \\
\hline & $70+$ & 7840 & $43.2(3385)$ \\
\hline \multirow[t]{5}{*}{ GO } & $<40$ & 848 & $1.3(11)$ \\
\hline & $40-49$ & 845 & $2.1(18)$ \\
\hline & $50-59$ & 984 & $6.4(63)$ \\
\hline & $60-69$ & 673 & $11.4(77)$ \\
\hline & $70+$ & 615 & 32.0 (197) \\
\hline
\end{tabular}

\begin{tabular}{|c|c|c|}
\hline & Controls & \\
\hline Number & $\begin{array}{c}5 \text {-year mortality risk (\%) } \\
\text { (number) }\end{array}$ & $\begin{array}{c}\text { Cases/controls } \\
\text { 5-year mortality ratio }\end{array}$ \\
\hline 27984 & $0.1(27)$ & 4 \\
\hline 18040 & $0.5(82)$ & 3 \\
\hline 19544 & $1.6(322)$ & 3.1 \\
\hline 16916 & $4.8(805)$ & 2.8 \\
\hline 31360 & $25.2(7909)$ & 1.7 \\
\hline 3392 & $0.1(2)$ & 13 \\
\hline 3380 & $0.4(15)$ & 5.3 \\
\hline 3936 & $1.9(75)$ & 3.4 \\
\hline 2692 & $4.1(110)$ & 2.8 \\
\hline 2460 & $19.9(490)$ & 1.7 \\
\hline
\end{tabular}

\section{Effect of gender and age}

Gender was profoundly related to mortality, both in GD and GO. Among GD patients, males had a significantly higher mortality than that in females (HR: 1.32 (1.25-1.40); $P<0.001$ and HR: 1.16 (1.12-1.19); $P<0.001$ respectively). In GO individuals, the relationship between gender and mortality was even more pronounced, with a HR of 1.52 $(1.30-1.78 ; P<0.001)$ in males and a HR of $1.10(0.98-1.23$; $P=0.119$ ) in females (Table 2). The influence of age on mortality in GD and GO is summarised in Table 3. Fiveyear mortality risk ratio did not change much over the decades for GD, but for GO, it was the highest in the youngest and decreased with increasing age.

\section{Difference between GO and GD}

The mortality risk in GO compared to GD was significantly decreased (HR: 0.64 (0.59-0.69); $P<0.001)$, although this effect declined after adjustment for pre-existing morbidity, age and gender (HR: $0.92(0.85-1.00) ; P=0.051)$. After restricting the analyses to GD cases alive and not lost to follow-up within 6 months after the thyroid diagnosis, results did not change significantly (HR (unadjusted): 0.75 (0.69-0.82); $P<0.001$ and HR (adjusted): 1.07 (0.99-1.16); $P=0.107)$.

\section{Discussion}

In this large-scale nationwide cohort study, comprising more than 30000 patients with Graves' disease, of whom nearly 4000 had GO, we found an $18 \%$ excess mortality in individuals with Graves' disease (GD+GO). This confirms our previous findings in a much smaller study population (1291 patients with GD) (6). More importantly, in the present study, we had ample power to investigate whether the mortality in GO differs from the background population and whether the mortality differs between GO and GD. Finally, we explored the influence of gender and age on mortality in patients with GD and GO.

The mortality in patients with GO has not previously been evaluated in detail and hitherto the limited knowledge is primarily based on case stories and a small case-control study $(18,19)$. These sparse data indicate an increased mortality in GO patients. However, they offer no evaluation of whether this could be due to GO per se, due to the burden of pre-existing morbidity, the treatment used for GO or whether the observed risk was higher than that in patients with GD. Here, we followed 3965 GO patients, over a median of 7.9 years and found a $23 \%$ increased mortality compared with an age- and gender-matched control population. The fact that the excess mortality remained significant after limiting the analyses to subjects without any pre-existing morbidity suggests that GO could be causally associated with increased mortality.

Our finding of a higher mortality in males than that in females is in line with existing knowledge on the manifestation and course of GD and GO. Thus, most (20, $21,22)$, albeit not all (23), have found a similar association between male gender and more severe disease and poorer prognosis. Indeed, it has been demonstrated that males with GD more frequently experience failure of medical treatment, are less likely to enter long-term remission (24) and have more severe GO $(18,19,20)$. The reason, perhaps, being poorer compliance and biochemical control, knowing that males are less frequent users of the healthcare system than are females (25). The untoward consequence of this may be that males are diagnosed and started on treatment at a later stage than females. 
We cannot qualify this statement as our databases do not incorporate data on thyroid function variables, effect of treatment or evaluation of compliance. Moreover, smoking is a known risk factor for developing orbitopathy in GD patients and for aggravation of the condition in GO patients (26). Although the current number of smokers in Denmark is nearly equally distributed between the genders, it was earlier very much dominated by males (Danish National Board of Health, www.sst.dk and the statistical bureau, www.dst.dk). For GD and GO appearing during 1995-2012, the relevant smoking exposure is decades earlier, at a time when smoking habits were skewed towards male dominance.

Less obvious are our findings from the age stratified analyses. Early onset of GD has previously been associated with a biochemically more severe disease and a poorer prognosis $(20,24)$. However, our study cannot verify that young GD patients have a higher mortality risk. In part, the explanation could be more intensive treatment of thyroid dysfunction in the young, as reflected by a higher referral rate of young patients to treatment in specialised units (27). In contrast, GO has been described to be more severe at late onset $(20,21,22,23,24)$, and yet, we found an inverse relationship between mortality and age. However, it is important to point out that the relative effect of mortality due to GO (as for any other disease) decreases when the probability of dying increases with age. In other words, the huge mortality ratio in the young is mainly due to the otherwise very low probability of dying at $<40$ years of age.

We hypothesised that patients with GO would have a higher mortality than that in patients with GD. However, unexpected and unexplained, we found a decreased mortality in GO compared to that in patients with GD, although the difference attenuated after adjustment for age, gender and pre-existing morbidity, indicating that the two phenotypes, despite the huge numbers investigated, do not with certainty differ significantly $(P=0.051)$ in mortality risk. In a EUGOGO survey investigating how initial management and presentation of GO has changed over time (28), it was demonstrated that during the last decade, patients are being diagnosed and referred earlier to tertiary centres and consequently treated more promptly. Perhaps early intervention and strict regulation of thyroid function are in part responsible for reducing mortality in GO compared to that in GD patients. Thus, as an example, mortality in hyperthyroid patients treated with radioiodine sufficient to induce hypothyroidism has been reported to reduce mortality to that of the background population (29). In our cohort, patients with GO may have been more likely to receive definitive treatment (thyroid surgery or radioactive iodine), and hence, rendered euthyroid (with or without thyroxine therapy) more quickly than patients with Graves' disease without eye involvement. Unfortunately, the effect of treatment modality on mortality cannot be evaluated further as the available data sources do not include this kind of information.

It is well accepted that smoking is associated with aggravation of and a poorer prognosis in GO (26). Importantly, counselling on smoking cessation has become central in the treatment of GO patients (30). If this intervention has been successful, it may also have contributed to a decline in mortality compared to that in patients with GD. Although treatment with steroids may be harmful (19), our finding of a similar mortality risk in GD and GO does not support that treatments given specifically to GO patients decreases life expectancy as compared to patients with GD.

The use of the Danish registers provided the possibility for large-scale investigation of a whole nation. Other strengths of our study include high power, the possibility of differentiating between Graves' disease patients with and without GO, the use of the standardised and validated CS to evaluate the degree of pre-existing morbidity as well as the relatively long follow-up time. On the other hand, there are also a number of limitations, including the risk of misclassification and the lack of detailed information on the severity of GO as well as lack of information on smoking habits, all of which deserve attention.

The registers used are robust and the DNPR, from which we identified our cases, has very high validity, with misclassification of thyroid diagnoses occurring in less than $2 \%$ of cases (15). Furthermore, we found the prevalence of GO in Graves' disease patients to be approximately $12 \%$ in our study population, which is in line with what has been reported in other cohorts of patients with Graves' disease (8). Accepting that the GD group may contain some erroneously diagnosed individuals (i.e. patients with toxic nodular goitre) and that the control population contains some individuals with undiagnosed or mild GD, our estimates of mortality as compared to the background population and of the difference in mortality between GO and GD are most likely underestimated. Furthermore, immortal time bias could lead to underestimation of the mortality risk in GO compared to that in GD (17). Often a GO diagnosis is preceded by a GD diagnosis. As a consequence, when comparing mortality in GO and GD, bias is possibly introduced as mortality cannot occur in the time between 
the GD and the GO diagnosis. However, as our results did not change after excluding GD individuals dead or lost to follow-up within 6 months after the thyroid diagnosis, immortal time bias is unlikely to have affected our results significantly.

For GO, there are many different classification systems, much based on subjective assessment of the patient, which makes the definition of GO somewhat arbitrary. Unfortunately, we are not able to further categorise the severity and/or activity of our GO cases. In the present study, all cases with GO have been evaluated and diagnosed within specialised endocrine and/or ophthalmologic units in a hospital setting. Clearly, this may introduce a bias towards a high prevalence of GO cases with moderateto-severe GO in our population because many cases with mild GO will not be referred to these specialised units. Therefore, as our GO population most likely comprises the patients with the gravest manifestations of eye involvement (and hyperthyroidism), we would expect a higher mortality risk in GO patients compared to that in GD patients. Another limitation in the comparison between GD and GO is the lack of information regarding the severity and duration of hyperthyroidism. As it may be expected that GO individuals, on average, have more severe hyperthyroidism, our finding of an equal mortality risk in subjects with GD and GO, further supports that a GO diagnosis is not associated with an increased mortality in Graves' disease patients.

As smoking plays an important role in the development and severity of GO and also increases mortality risk, this lack of information could bias our findings. However, adjusting for the use of drugs for obstructive airway diseases, a disorder very firmly linked to smoking, our findings are, at least partly, adjusted for the possible effect of smoking. Again, as most GO patients are current or previous smokers, this ought to have rendered them at higher risk of dying, thereby, in fact, strengthening our findings.

Future studies should focus on the importance of smoking habits, type and effect of treatment, influence of referral to tertiary centres, comorbidity, work disability and cause of death, to clarify the underlying reasons for the association between gender, age and mortality, and the lack of excess mortality in GO compared to GD. In conclusion, our study provides robust evidence from population-based, nationwide data of both GD and GO being associated with an increased mortality. We show that this excess mortality is influenced by gender, age and burden of pre-existing morbidity. Surprisingly, and in need of further study, a GO diagnosis is not associated with an increased mortality in Graves' disease patients.

\section{Declaration of interest}

The authors declare that there is no conflict of interest that could be perceived as prejudicing the impartiality of this study.

\section{Funding}

The Danish thyroid patient organisation, 'Musikforlæggerne Agnes and Knut Mørk's Foundation', and a pre-graduate grant from Odense University Hospital to Charlotte F Schwensen. Research grant from the European Thyroid Association to Frans Brandt.

\section{Author contribution statement}

Laszlo Hegedüs and Thomas $\mathrm{H}$ Brix designed the study. Charlotte $\mathrm{F}$ Schwensen and Frans Brandt collected and analysed the data. Charlotte F Schwensen wrote the first draft of this report. All authors made critical revisions of the manuscript.

\section{Acknowledgements}

The collection of data was funded by donations from (1) The Danish thyroid patient organisation, (2) Agnes and Knut Mørk's Foundation and (3) The European Thyroid Association.

\section{References}

1 Smith T \& Hegedüs L. Graves' disease. New England Journal of Medicine 2016375 1552-1565. (doi:10.1056/NEJMra1510030)

2 Brandt F, Thvilum M, Almind D, Christensen K, Green A, Hegedüs L \& Brix TH. Morbidity before and after the diagnosis of hyperthyroidism: a nationwide register-based study. PLOS ONE 20138 e66711. (doi:10.1371/journal.pone.0066711)

3 Brandt F, Thvilum M, Almind D, Christensen K, Green A, Hegedüs L \& Brix TH. Hyperthyroidism and psychiatric morbidity: evidence from a Danish nationwide register study. European Journal of Endocrinology 2014170 341-348. (doi:10.1530/EJE-13-0708)

4 Brandt F, Thvilum M, Hegedüs L \& Brix TH. Hyperthyroidism is associated with work disability and loss of labour market income. A Danish register-based study in singletons and disease-discordant twin pairs. European Journal of Endocrinology 2015173 595-602. (doi:10.1530/EJE-15-0306)

5 Brandt F, Green A, Hegedüs L \& Brix TH. A critical review and meta-analysis of the association between overt hyperthyroidism and mortality. European Journal of Endocrinology 2011165 491-497. (doi:10.1530/EJE-11-0299)

6 Brandt F, Thvilum M, Almind D, Christensen K, Green A, Hegedüs L \& Brix TH. Graves' disease and toxic nodular goiter are both associated with increased mortality but differ with respect to the cause of death: a Danish population-based register study. Thyroid 2013 23 408-413. (doi:10.1089/thy.2012.0500)

7 Tanda ML, Piantanida E, Liparulo L, Veronesi G, Lai A, Sassi L, Pariani N, Gallo D, Azzolini C, Ferrario M \& Bartalena L. Prevalence and natural history of Graves' orbitopathy in a large series of patients with newly diagnosed Graves' hyperthyroidism seen at a single center. Journal of Clinical Endocrinology and Metabolism 201398 1443-1449. (doi:10.1210/jc.2012-3873)

8 Laurberg P, Berman DC, Pedersen IB, Andersen S \& Carlé A. Incidence and clinical presentation of moderate to severe Graves' orbitopathy 
in a Danish population before and after iodine fortification of salt. Journal of Clinical Endocrinology and Metabolism 201297 2325-2332. (doi:10.1210/jc.2012-1275)

9 Ponto KA, Merkesdal S, Hommel G, Pitz S, Pfeiffer N \& Kahaly GJ. Public health relevance of Graves' orbitopathy. Journal of Clinical Endocrinology and Metabolism 201398 145-152. (doi:10.1210/jc.20123119)

10 Wiersinga WM. Quality of life in Graves' ophthalmopathy. Best Practice and Research Clinical Endocrinology and Metabolism 201226 359-370. (doi:10.1016/j.beem.2011.11.001)

11 Kahaly GJ, Petrak F, Hardt J, Pitz S \& Egle UT. Psychosocial morbidity of Graves' orbitopathy. Clinical Endocrinology 200563 395-402. (doi:10.1111/j.1365-2265.2005.02352.x)

12 Thygesen LC, Daasnes C, Thaulow I \& Brønnum-Hansen H. Introduction to Danish (nationwide) registers on health and social issues: structure, access, legislation, and archiving. Scandinavian Journal of Public Health 201139 (Supplement 7) 12-16. (doi:10.1177/1403494811399956)

13 Pedersen CB. The Danish civil registration system. Scandinavian Journal of Public Health 201139 (Supplement 7) 22-25. (doi:10.1177/1403494810387965)

14 Lynge E, Sandegaard JL \& Rebolj M. The Danish National Patient Register. Scandinavian Journal of Public Health 201139 (Supplement 7) 30-33. (doi:10.1177/1403494811401482)

15 Kildemoes HW, Sørensen HT \& Hallas J. The Danish National Prescription Registry. Scandinavian Journal of Public Health 201139 (Supplement 7) 38-41. (doi:10.1177/1403494810394717)

16 Tang J, Wan JY \& Bailey JE. Performance of comorbidity measures to predict stroke and death in a community-dwelling, hypertensive Medicaid population. Stroke 200839 1938-1944. (doi:10.1161/ STROKEAHA.107.504688)

17 Suissa S. Immortal time bias in pharmaco-epidemiology. American Journal of Epidemiology 200815 492-499. (doi:10.1093/aje/kwm324)

18 Schaefer U, Hesselmann S, Micke O, Schueller P, Bruns F, Palma C \& Willich N. A long-term follow-up study after retro-orbital irradiation for Graves' ophthalmopathy. International Journal of Radiation Oncology Biology Physics 200252 192-197. (doi:10.1016/S03603016(01)01754-0)

19 Marcocci C, Watt T, Altea MA, Rasmussen AK, Orgiazzi J \& Bratalena L. Fatal and non-fatal adverse events of glucocorticoid therapy for Graves' orbitopathy: a questionnaire survey among members of the European Thyroid Association. European Journal of Endocrinology 2012166 247-253. (doi:10.1530/EJE-11-0779)

20 Manji N, Carr-Smith JD, Boelaert K, Allahabadia A, Armitage M, Chatterjee VK, Lazarus JH, Pearce SH, Vaidya B, Gough SC et al.
Influences of age, gender, smoking, and family history on autoimmune thyroid disease phenotype. Journal of Clinical Endocrinology and Metabolism 200691 4873-4880. (doi:10.1210/ jc.2006-1402)

21 Kendler DL, Lippa J \& Rootman J. The initial clinical characteristics of Graves' orbitopathy vary with age and sex. Archives of Ophthalmology 1993111 197-201. (doi:10.1001/archopht.1993.01090020051022)

22 Perros P, Crombie AL, Matthews JN \& Kendall-Taylor P. Age and gender influence the severity of thyroid-associated ophthalmopathy: a study of 101 patients attending a combined thyroid-eye clinic. Clinical Endocrinology 199338 367-372. (doi:10.1111/j.1365-2265.1993.tb00516.x)

23 Khong JJ, Finch S, De Silva C, Rylander S, Craig JE, Selva D \& Ebeling PR. Risk factors for Graves' orbitopathy; the Australian Thyroid-associated Orbitopathy Research (ATOR) study. Journal of Clinical Endocrinology and Metabolism 2016101 2711-2720. (doi:10.1210/jc.2015-4294)

24 Allahabadia A, Daykin J, Holder RL, Sheppard MC, Gough SCL \& Franklyn JA. Age and gender predict the outcome of treatment for Graves' hyperthyroidism. Journal of Clinical Endocrinology and Metabolism 200085 1038-1042. (doi:10.1210/jc.85.3.1038)

25 Koopmans GT \& Lamers LM. Gender and health care utilization: the role of mental distress and help-seeking propensity. Social Science and Medicine 200764 1216-1230. (doi:10.1016/j.socscimed.2006.11.018)

26 Hegedüs L, Brix TH \& Vestergaard P. Relationship between cigarette smoking and Graves' ophthalmopathy. Journal of Endocrinological Investigation 200427 265-271. (doi:10.1007/bf03345276)

27 Carlé A, Pedersen IB, Perrild H, Ovesen L, Jørgensen T \& Laurberg P. High age predicts low referral of hyperthyroid patients to specialized hospital departments: evidence for referral bias. Thyroid 201323 1518-1524. (doi:10.1089/thy.2013.0074)

28 Perros P, Zarkovic M, Azzolini C, Ayvaz G, Baldeschi L, Bartalena L, Boschi A, Bournaud C, Brix TH, Covelli D et al. PREGO (presentation of Graves' orbitopathy) study: changes in referral patterns to European Group On Graves' Orbitopathy (EUGOGO) centres over the period from 2000 to 2012. Britisch Journal of Ophthalmology 201599 1531-1535. (doi:10.1136/bjophthalmol-2015-306733)

29 Franklyn JA, Sheppard MC \& Maisonneuve P. Thyroid function and mortality in patients treated for hyperthyroidism. JAMA 2005294 71-80. (doi:10.1001/jama.294.1.71)

30 Bartalena L, Baldeschi L, Boboridis K, Eckstein A,Kahaly GJ, Marococci C, Perros P, Salvi M \& Wiersinga WM. The 2016 European Thyroid Association/European Group on Graves' orbitopathy guidelines for the management of Graves' orbitopathy. European Thyroid Journal 20165 9-26. (doi:10.1159/000443828)

Received 21 November 2016

Revised version received 25 February 2017

Accepted 3 March 2017 